\title{
Asymptomatic obstructive hydronephrosis associated with diabetes insipidus: a case report and review
}

\author{
Long Sun, Dongyan Zhao, Linfeng Zhu, Yiding Shen, Yijun Zhao, Daxing Tang \\ Department of Urology, Children's Hospital, Zhejiang University School of Medicine, National Clinical Research Center for Child Health, \\ Hangzhou, China \\ Correspondence to: Daxing Tang. Department of Urology, Children's Hospital, Zhejiang University School of Medicine, No. 57 of Zhugan Lane, \\ Hangzhou 310003, China. Email: tangdx0206@zju.edu.cn.
}

\begin{abstract}
The treatment of asymptomatic hydronephrosis due to ureteropelvic junction obstruction (UPJO), as well as the timing and indications for surgical intervention, remain controversial. Diabetes insipidus (DI) is a rare disease in infants that is known to cause non-obstructive hydronephrosis, while its association with obstructive hydronephrosis has not been reported. Some studies have found that increased water intake is a predisposing risk factor of developing hydronephrosis. However, there are no studies or guidelines that indicate the specific follow-up intervals and surgical indications for mild hydronephrosis if predisposing risk factors persist. A 46-month-old boy was admitted to our Urology Department with a history of Society of Fetal Urology (SFU) Grade 2 prenatal left hydronephrosis, which was stable at SFU Grade 1-2 at regular postnatal follow-ups. The patient developed polydipsia and polyuria three months prior to admission, then he was considered as primary polydipsia by endocrinology and was treated with fluid restriction while the examination was negative. Renal ultrasound at follow-up demonstrated severe left hydronephrosis with an anterior-posterior diameter (APD) of $6.25 \mathrm{~cm}$ three months after symptom onset. Diuretic renography (DR) revealed a renal function of $13.7 \%$ with a glomerular filtration rate (GFR) of $11.25 \mathrm{~mL} / \mathrm{min}$. The patient was otherwise asymptomatic without any abdominal pain or vomiting. He underwent left pyelostomy immediately as well as laparoscopic left dismembered ureteropelvioplasty after three months, and a diagnosis of UPJO was confirmed. The patient had an uncomplicated postoperative recovery and the result of follow-up renal ultrasound was stable. However, the symptoms of polydipsia and polyuria did not improve significantly. He underwent pituitary magnetic resonance imaging (MRI) and pathological examination, the results of which were consistent with central DI caused by Langerhans cell hyperplasia four months postoperatively. This case indicates the need to pay attention to children with mild hydronephrosis undergoing regular observation and conservative treatment, as a sudden aggravation of the hydronephrosis and a rapid decline of renal function may occur if DI persists.
\end{abstract}

Keywords: Asymptomatic hydronephrosis; ureteropelvic junction obstruction (UPJO); diabetes insipidus (DI); case report

Submitted Dec 25, 2020. Accepted for publication Apr 20, 2021.

doi: $10.21037 / \mathrm{tp}-20-476$

View this article at: http://dx.doi.org/10.21037/tp-20-476

\section{Introduction}

Prenatal ultrasound has become a part of routine prenatal care since the 1980s and the diagnostic frequency of fetal hydronephrosis has increased significantly since then (1). Most infants with fetal hydronephrosis are asymptomatic at birth and as time goes on the hydronephrosis can resolve, progress or remain stable (2). There is no clear consensus on the management of asymptomatic hydronephrosis (3) as asymptomatic hydronephrosis can improve on its own without intervention. It remains controversial that whether surgical intervention at an early stage is necessary in order 

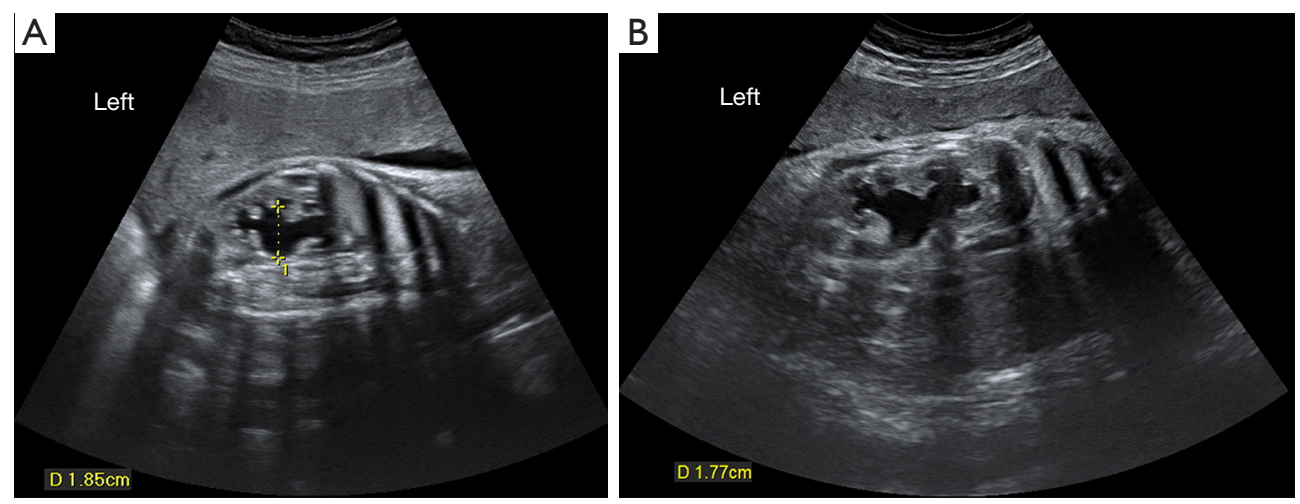

Figure 1 Prenatal ultrasound. (A) Left hydronephrosis was first identified at 30 weeks of pregnancy. (B) Left hydronephrosis at 37 weeks of pregnancy.

to avoid renal function decline (4).

Diabetes insipidus (DI) (5) is a syndrome characterized by hypotonic polyuria and polydipsia with an incidence of 1 in 25,000 . The most common type is central DI, which occurs due to inadequate secretion or deficient synthesis of arginine vasopressin caused by osmotic stimulation of the hypothalamus-neurohypophyseal system (6). DI is often difficult to diagnose reliably and accurately, particularly among patients with primary polydipsia or some mild forms of central DI. Some studies have found that increased water intake is a predisposing risk factor of developing hydronephrosis (7). However, there are no studies or guidelines that indicate the specific follow-up intervals and surgical indications for mild hydronephrosis if predisposing risk factors persist. DI causes non-obstructive hydronephrosis (8); however, to the best of our knowledge, no studies have shown whether DI can cause or aggravate obstructive hydronephrosis. This article describes a case of asymptomatic obstructive hydronephrosis that was aggravated by DI. We present the following case in accordance with the CARE reporting checklist (available at http://dx.doi.org/10.21037/tp-20-476).

\section{Case presentation}

A 46-month-old boy was found to have left hydronephrosis on ultrasound at 30 weeks of pregnancy (Figure 1). He was followed up regularly with renal ultrasound after birth and the anterior-posterior diameter (APD) of hydronephrotic renal pelvis was stable between 1 and $2.5 \mathrm{~cm}$ (Figure $2 A$ ). The patient started to have compulsive water intake more than $3 \mathrm{~L} / \mathrm{d}$ three months prior to admission, as well as polydipsia and polyuria. He was admitted to the Department of Endocrinology at our hospital one month after symptom onset and a repeated renal ultrasound revealed an APD of $2.3 \mathrm{~cm}$. The patient underwent water deprivation test and the result showed that the urine output decreased from 6.55 to $4.90 \mathrm{~mL} / \mathrm{kg} / \mathrm{h}$, and urinary osmolality was $298 \mathrm{mOsm} / \mathrm{kg}$ with no significant change after desmopressin injection. A diagnosis of primary polydipsia was considered at that time (5). Water restriction treatment with approximately $2 \mathrm{~L}$ of water per day was initiated. Two months after treatment, the patient was found to have significantly worsening left hydronephrosis with a renal pelvis APD of $6.25 \mathrm{~cm}$ on the follow-up renal ultrasound (Figure $2 \mathrm{~B}$ ). The patient was otherwise asymptomatic without any abdominal pain or vomiting. On review of the patient's past medical history, there were no urinary symptoms in the first 3 years, no nocturia and drinking during the night, and no psychiatric comorbidities or medications. Body weight (15.5 $\mathrm{kg})$, height $(97 \mathrm{~cm})$, blood pressure $(120 / 73 \mathrm{mmHg})$, and pulse (116/min). Normal abdominal exam. Renal function, serum and urine electrolyte levels were within normal limits. Urine specific gravity was 1.008. Renal ultrasound was done after three months of polyuria and polydipsia, which showed severe hydronephrosis (Figure 2B) and this was re-demonstrated on magnetic resonance urography (Figure 2C). There was no obvious abnormality on the pituitary magnetic resonance imaging (MRI). Diuretic renography (DR) showed a glomerular filtration rate (GFR) of $11.25 \mathrm{~mL} / \mathrm{min}$ in the left kidney, which indicated $13.7 \%$ split renal function, while the right side was $70.86 \mathrm{~mL} / \mathrm{min}$.

Pyelostomy was performed immediately as the hydronephrosis significantly aggravated and the renal function significantly declined in a short period without a clear etiology. After three months, there was no obvious 

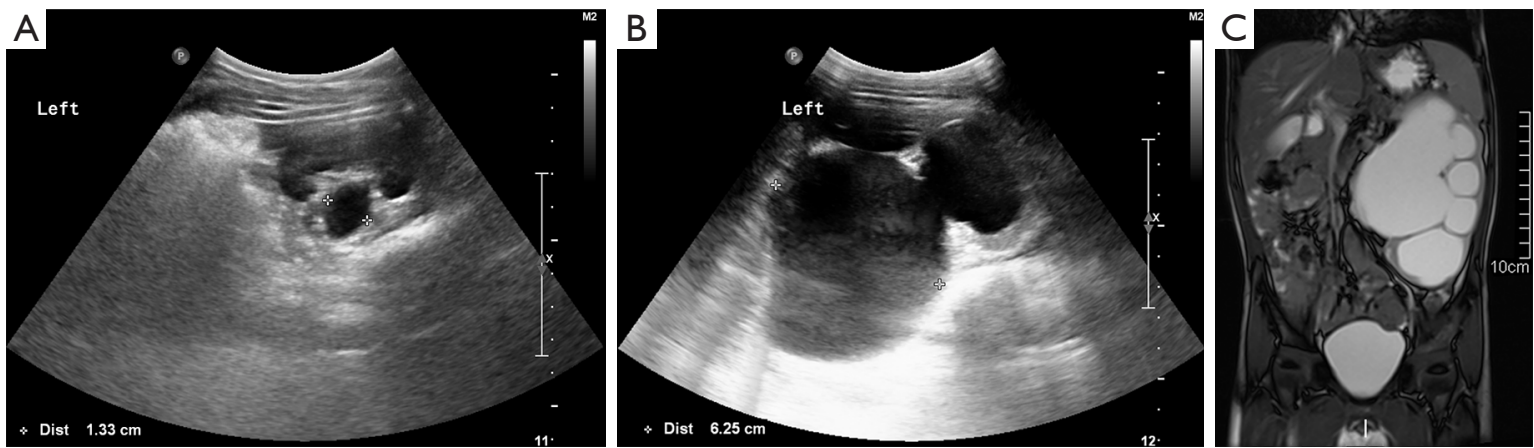

Figure 2 Hydronephrosis changes on imaging examinations before admission. (A) The APD of renal pelvis was between 1 and $2.5 \mathrm{~cm}$ after birth. (B) One week before presentation, the hydronephrosis was aggravated with a renal pelvis APD of $6.25 \mathrm{~cm}$. (C) Magnetic resonance urography showed severe left hydronephrosis. APD, anterior-posterior diameter.
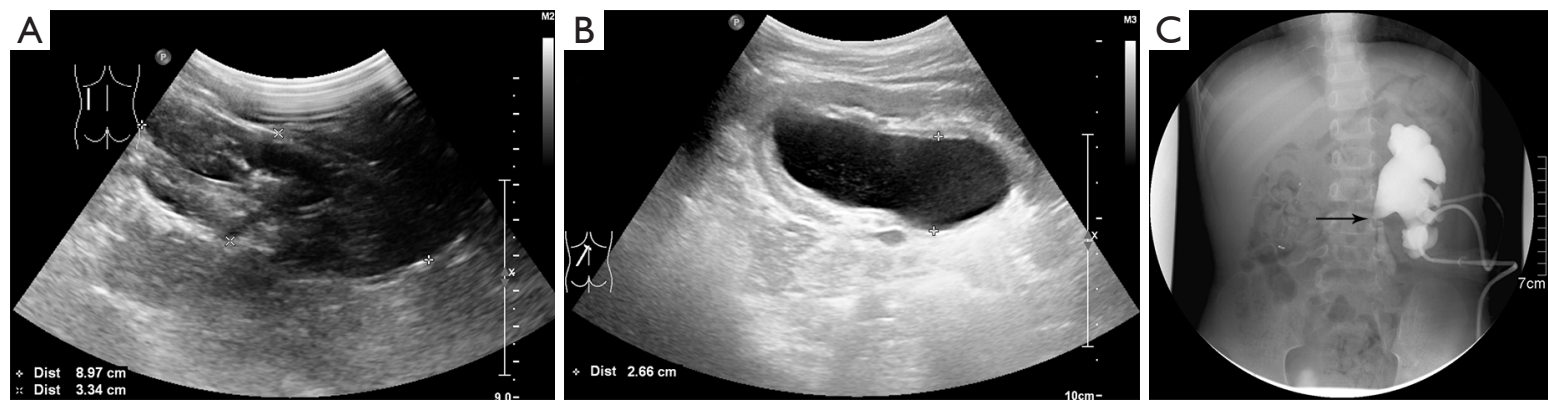

Figure 3 Imaging examinations after pyelostomy. (A) Three months after pyelostomy, there was no obvious dilation of the renal pelvis on ultrasound. (B) Four hours after clipping the fistula, the renal pelvis APD increased to $2.7 \mathrm{~cm}$ on ultrasound. (C) UPJO was suspected on antegrade pyelography (black arrow). APD, anterior-posterior diameter; UJPO, ureteropelvic junction obstruction.

dilation of the renal pelvis on ultrasound (Figure $3 A$ ). Ultrasound revealed a renal pelvis APD of $2.7 \mathrm{~cm}$ after clipping the fistula for four hours (Figure $3 B$ ). Ureteropelvic junction obstruction (UPJO) was suspected, consistent with the preoperative results of antegrade pyelography (Figure 3C). Laparoscopic left dismembered ureteropelvioplasty was performed with parents' agreement and showed UPJO (Figure 4A). Postoperative pathological examination showed chronic inflammation of the ureteral mucosa.

The patient had an uncomplicated postoperative recovery. Post-operative renal function, serum and urine electrolyte levels remained stable, renal ultrasound showed a renal pelvis APD between 2.4 and $2.7 \mathrm{~cm}$ during three months after laparoscopic operation (Figure $4 B$ ), while the symptoms of polydipsia and polyuria did not improve significantly. Urine output was $6.35 \mathrm{~mL} / \mathrm{kg} / \mathrm{h}$ and urinary osmolality was $301 \mathrm{mOsm} / \mathrm{kg}$. Repeated pituitary MRI showed pituitary stalk thickening and cranial computed tomography showed bone destruction. A bone biopsy revealed fibrous adipose tissue and focal inflammatory cell infiltration. A bone marrow biopsy showed hypercellular. The results were consistent with central DI caused by Langerhans cell hyperplasia four months postoperatively. Treatment was initiated with desmopressin, glucocorticoid and chemotherapy and his polydipsia improved (about 1-1.5 L/d; Table 1).

All procedures performed in studies involving human participants were in accordance with the ethical standards of the institutional and/or national research committee(s) and with the Helsinki Declaration (as revised in 2013). Written informed consent was obtained from the patient.

\section{Discussion}

Prenatal ultrasound has been increasingly implemented in prenatal screening and more than $90 \%$ of pregnant women receive it. Approximately 70\% of neonates diagnosed with 

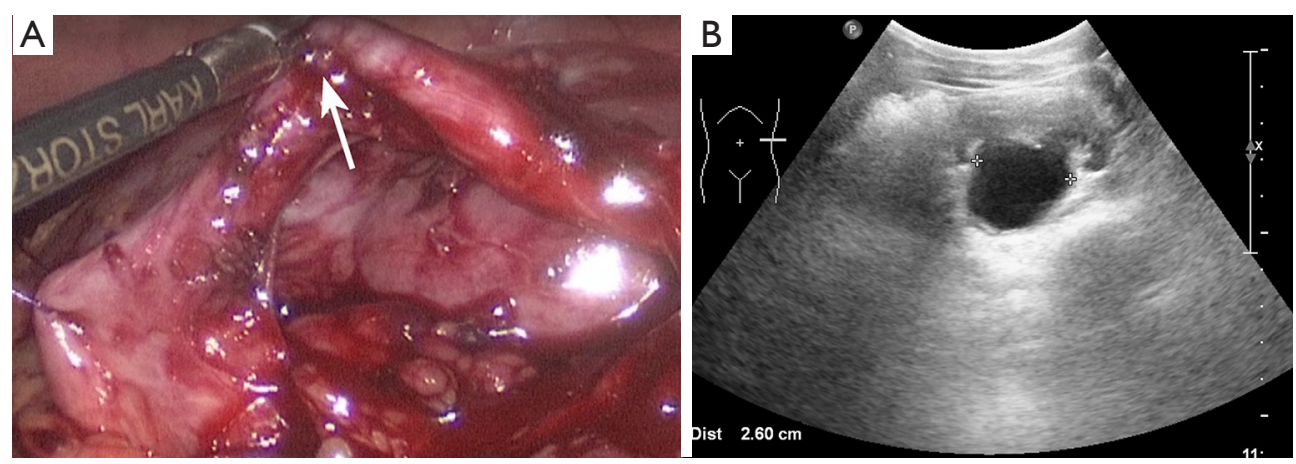

Figure 4 Findings in laparoscopy and postoperative ultrasound. (A) UPJO was confirmed intraoperatively (white arrow). (B) Ultrasound showed a renal pelvis APD of $2.6 \mathrm{~cm}$ two months postoperatively. APD, anterior-posterior diameter; UJPO, ureteropelvic junction obstruction.

Table 1 The timeline of patient's diagnosis and treatment

\begin{tabular}{|c|c|c|c|c|}
\hline Timeline & APD (cm) & Polydipsia & Diagnosis & Treatment \\
\hline-90 days & 2.1 & About $3 \mathrm{~L} / \mathrm{d}$ & Mild hydronephrosis & Regular follow-up \\
\hline-60 days & 2.3 & About $2 \mathrm{~L} / \mathrm{d}$ & $\begin{array}{l}\text { Mild hydronephrosis; Primary } \\
\text { polydipsia }\end{array}$ & Water restriction \\
\hline 90 days & 2.7 & About $2 \mathrm{~L} / \mathrm{d}$ & UPJO; primary polydipsia & $\begin{array}{l}\text { Laparoscopic dismembered } \\
\text { ureteropelvioplasty; water } \\
\text { restriction }\end{array}$ \\
\hline 210 days & 2.4 & About $1-1.5 \mathrm{~L} / \mathrm{d}$ & UPJO; central diabetes insipidus & $\begin{array}{l}\text { Desmopressin; glucocorticoid; } \\
\text { chemotherapy }\end{array}$ \\
\hline
\end{tabular}

"-" means the time of before admission. APD, anterior-posterior diameter. UJPO, ureteropelvic junction obstruction.

prenatal hydronephrosis do not have clinical symptoms or signs (3). The identification of these asymptomatic prenatal hydronephrosis may minimize renal damage with regular follow-up. Mild hydronephrosis may be transitional and can resolve spontaneously; however, delayed intervention for obstructive hydronephrosis could affect the postoperative recovery of renal function (9). The suitable time and exact indications for surgical intervention are complex and remain controversial (4). Over the past few decades, there has been a trend toward choosing conservative treatment for asymptomatic hydronephrosis (1); therefore, regular followup is crucial to prevent renal dysfunction in this patient population.

Shapiro et al. (10) reported the association between hydronephrosis and DI in 1978 and it is recommended that patients should undergo serial intravenous pyelograms and renal function assessments if polyuria persists, and surgery should be reserved for cases with evidence of obstruction. In the 1980 s, the diagnostic rate of asymptomatic hydronephrosis increased significantly with the advent of fetal ultrasonography (3). As asymptomatic hydronephrosis may improve spontaneously without intervention, initial over aggressive intervention was tempered. Renal pelvis APD and split renal function below $40 \%$ have been considered the main parameters to indicate surgical intervention. In 1993, Society for Fetal Urology (SFU) proposed the importance of the thinning of the parenchyma as it could lead to decrease of the renal function (11). And some scholars have recently proposed follow-up intervals for different grades of hydronephrosis (12). It is recommended to repeat ultrasound every 3-6 months for SFU grade 1-2 asymptomatic hydronephrosis $(12,13)$. More 
than $80 \%$ of these patients can experience spontaneous resolution of hydronephrosis within one year (14); if hydronephrosis persists, repeat ultrasonography every 6 months is recommended. For patients with SFU grade 3 hydronephrosis, ultrasound performed every 3 months is recommended and further DR examination should be performed if there is progression of hydronephrosis (12). Further DR is necessary for SFU grade 4 hydronephrosis, while surgical treatment may be a better choice than routine follow-up (15). The indications for surgical intervention include: deterioration of hydronephrosis, decline of split renal function $>10 \%$, split renal function $<40 \%$, obstruction curve on DR, or febrile urinary tract infection (12). It is worth noting that hydronephrosis and/ or deterioration of renal function may recur a few years after spontaneous resolution of hydronephrosis, and it is usually accompanied by symptoms, such as abdominal pain, nausea, vomiting, and hematuria (16). Carpenter et al. (8) reported that patients should undergo continuous careful monitoring postoperatively if symptoms persist, such as polydipsia and polyuria. The clinical/surgical approach has changed a lot and plays a positive role in the preservation of renal function. Hydronephrosis was identified by prenatal ultrasound in this case, and there was no obvious change in the size of the hydronephrosis and the patient remained asymptomatic during the follow-up over 3 years. After experiencing polydipsia (water intake $>3 \mathrm{~L} / \mathrm{d}$ ) for one month, a slight progression of hydronephrosis was observed. The hydronephrosis further progressed significantly without additional symptoms after water intake control for 2 months, during which he was shown to have a renal pelvis APD of more than $6 \mathrm{~cm}$ on ultrasound and a split renal function of $13.7 \%$ on DR. We discuss the features of this special case as below.

The patient had severe hydronephrosis but no acute obstructive symptoms. The manifestations of acute obstructive hydronephrosis include flank pain or lower back pain with or without accompanying nausea and vomiting, and young patients can have periumbilical pain or upper abdominal pain instead. Gross or microscopic hematuria, which occurs secondary to vascular rupture due to the rapid expansion of renal collecting system, is also common (7). This 46-month-old boy was found to have asymptomatic SFU grade 1-2 hydronephrosis on prenatal ultrasound and he was undergoing follow-up ultrasound every 6 months since 1-year old. The follow-up interval was shortened as the patient started to have polydipsia and he was found to have worsening hydronephrosis and renal function after
3 months. After taking a detailed medical history, we found an uncommon phenomenon in that the patient had no other symptoms such as abdominal pain, nausea, vomiting, hematuria, unexplained fever and urinary tract infection but polydipsia and polyuria. Only a few studies showed that giant hydronephrosis could be asymptomatic until the late stage (17), or only manifests with non-specific symptoms (18), such as fatigue or indigestion. The incidence of asymptomatic hydronephrosis among newborns is $0.5 \%$ to $1 \%(19)$, and the incidence of central DI is 3-4 patients per 100,000 (20). As asymptomatic hydronephrosis is more common, we should pay special attention to it when it is accompanied by DI, other nephrologic disorders or symptoms (increased water intake, strenuous exercise or urinary retention). In this case, the patient did not report any symptoms and this could have affected prompt diagnosis and treatment. It is possible that the patient was not able to describe symptoms due to his young age, rather than asymptomatic. Some studies have found that increased water intake is a predisposing risk factor of developing hydronephrosis (7). However, to the best of our knowledge, there are no studies or guidelines that indicate the specific follow-up intervals and surgical indications for mild hydronephrosis if predisposing risk factors persist. For the purpose of monitoring hydronephrosis and protecting renal function, follow-up ultrasound performed every 1-2 weeks may be appropriate for patients with asymptomatic SFU grade 1-2 hydronephrosis if there are any factors that may lead to the exacerbation of hydronephrosis, as clinical symptoms may be inconsistent with imaging findings in these patients. Furthermore, even if there is no obvious change of hydronephrosis on ultrasound at the early stage, we recommend close follow-up of these patients as a sudden aggravation of hydronephrosis and rapid decline of renal function may occur at a later stage. In addition, we should always look for concurrent nephrologic disorders among patients with asymptomatic hydronephrosis. For example, the co-existence of vesicoureteral reflux (VUR) was found in $25 \%$ of newborns with asymptomatic hydronephrosis and it can lead to kidney damage with scar formation and a decrease of the renal function (21). Therefore, we should pay more attention if patients have symptoms, such as fever, turbid urine or ureteral dilatation on ultrasonography. Further examinations, such as urine analysis and voiding cystourethrogram, should be performed to rule out the coexistence of VUR.

Does central DI cause or aggravate obstructive hydronephrosis? In 1892, Osler (22) first described long-term 
polyuria induced bilateral non-obstructive hydronephrosis. Present literatures show that hydronephrosis is mostly associated with renal DI, while central DI is relatively rare. In 1980, Boyd et al. (23) reported a case of hydronephrosis associated with central DI, characterized by dilatation of bilateral urinary tract and bilateral non-obstructive hydronephrosis. From then on, several related reports have been published $(24,25)$. There is few literature about the correlation between DI and obstructive hydronephrosis. Giant hydronephrosis is a chronic progressive disease but there is no literature specifically describing how long this process takes. It was difficult to estimate when hydronephrosis started or was aggravated due to a long history in those above-mentioned cases. Therefore, we hypothesize that it takes a long time to develop nonobstructive hydronephrosis in patients with DI, may be 1 year or several years. This process may be shorter in patients with obstructive hydronephrosis. For example, hydronephrosis was significantly aggravated in 3 months or probably even shorter in this case. Therefore, further research is needed to determine how soon DI should be controlled and whether surgical intervention is necessary to preserve the renal function before hydronephrosis progresses.

\section{Conclusions}

Attention should be paid to children with mild hydronephrosis undergoing regular observation and conservative treatment as a sudden aggravation of hydronephrosis and rapid decline of renal function may occur if DI persists.

\section{Acknowledgments}

We would like to thank Editage (www.editage.cn) for English language editing.

Funding: None.

\section{Footnote}

Reporting Checklist: The authors have completed the CARE reporting checklist. Available at http://dx.doi.org/10.21037/ tp-20-476

Peer Review File: Available at http://dx.doi.org/10.21037/tp$20-476$

Conflicts of Interest: All authors have completed the ICMJE uniform disclosure form (available at http://dx.doi. org/10.21037/tp-20-476). The authors have no conflicts of interest to declare.

Ethical Statement: The authors are accountable for all aspects of the work in ensuring that questions related to the accuracy or integrity of any part of the work are appropriately investigated and resolved. All procedures performed in studies involving human participants were in accordance with the ethical standards of the institutional and/or national research committee(s) and with the Helsinki Declaration (as revised in 2013). Written informed consent was obtained from the patient.

Open Access Statement: This is an Open Access article distributed in accordance with the Creative Commons Attribution-NonCommercial-NoDerivs 4.0 International License (CC BY-NC-ND 4.0), which permits the noncommercial replication and distribution of the article with the strict proviso that no changes or edits are made and the original work is properly cited (including links to both the formal publication through the relevant DOI and the license). See: https://creativecommons.org/licenses/by-nc-nd/4.0/.

\section{References}

1. Yamaçake KG, Nguyen HT. Current management of antenatal hydronephrosis. Pediatr Nephrol 2013;28:237-43.

2. Menon P, Rao KL, Sodhi KS, et al. Hydronephrosis: Comparison of extrinsic vessel versus intrinsic ureteropelvic junction obstruction groups and a plea against the vascular hitch procedure. J Pediatr Urol 2015;11:80.e1-6.

3. Kohno M, Ogawa T, Kojima Y, et al. Pediatric congenital hydronephrosis (ureteropelvic junction obstruction): Medical management guide. Int J Urol 2020;27:369-76.

4. Ulman I, Jayanthi VR, Koff SA. The long-term followup of newborns with severe unilateral hydronephrosis initially treated nonoperatively. J Urol 2000;164:1101-5.

5. Christ-Crain M, Bichet DG, Fenske WK, et al. Diabetes insipidus. Nat Rev Dis Primers 2019;5:54.

6. Babey M, Kopp P, Robertson GL. Familial forms of diabetes insipidus: clinical and molecular characteristics. Nat Rev Endocrinol 2011;7:701-14.

7. Tsai JD, Huang FY, Lin CC, et al. Intermittent hydronephrosis secondary to ureteropelvic junction obstruction: clinical and imaging features. Pediatrics 2006;117:139-46.

8. Carpenter CP, Rawson A, Hains DS, et al. Resolution of 
Diabetes Insipidus After Pyeloplasty: A Case Report and Review of the Literature. Urology 2018;115:168-70.

9. Blachar A, Blachar Y, Livne PM, et al. Clinical outcome and follow-up of prenatal hydronephrosis. Pediatr Nephrol 1994;8:30-5.

10. Shapiro SR, Woerner S, Adelman RD, et al. Diabetes insipidus and hydronephrosis. J Urol 1978;119:715-9.

11. Fernbach SK, Maizels M, Conway JJ. Ultrasound grading of hydronephrosis: introduction to the system used by the Society for Fetal Urology. Pediatr Radiol 1993;23:478-80.

12. Capolicchio JP, Braga LH, Szymanski KM. Canadian Urological Association/Pediatric Urologists of Canada guideline on the investigation and management of antenatally detected hydronephrosis. Can Urol Assoc J 2018;12:85-92.

13. Chertin B, Pollack A, Koulikov D, et al. Conservative treatment of ureteropelvic junction obstruction in children with antenatal diagnosis of hydronephrosis: lessons learned after 16 years of follow-up. Eur Urol 2006;49:734-8.

14. Alconcher LF, Tombesi MM. Natural history of bilateral mild isolated antenatal hydronephrosis conservatively managed. Pediatr Nephrol 2012;27:1119-23.

15. Ross SS, Kardos S, Krill A, et al. Observation of infants with SFU grades 3-4 hydronephrosis: worsening drainage with serial diuresis renography indicates surgical intervention and helps prevent loss of renal function. J Pediatr Urol 2011;7:266-71.

16. Matsui F, Shimada K, Matsumoto F, et al. Late recurrence of symptomatic hydronephrosis in patients with prenatally detected hydronephrosis and spontaneous improvement. J Urol 2008;180:322-5.

17. Ardiçoğlu A, Yüzgeç V, Atikeler MK, et al. A case of adult giant hydronephrosis as unusual cause of intraabdominal mass. Int Urol Nephrol 2003;35:7-8.

18. Budigi B, Dyer RB. "Giant" hydronephrosis. Abdom Radiol (NY) 2019;44:1946-8.

19. Burgu B, Aydogdu O, Soygur T, et al. When is it necessary to perform nuclear renogram in patients with a unilateral neonatal hydronephrosis? World J Urol 2012;30:347-352.

20. Patti G, Ibba A, Morana G, et al. Central diabetes insipidus in children: Diagnosis and management. Best Pract Res Clin Endocrinol Metab 2020;34:101440.

21. Radmayr C, Bogaert G, Dogan HS, et al. EAU Guidelines on Paediatric. Urology 2020:59-62.

22. Osler $W$. The principles and practice of medicine: designed for the use of practitioners and students of medicine. 1st ed. New York: D. Appleton, 1892.

23. Boyd SD, Raz S, Ehrlich RM. Diabetes insipidus and nonobstructive dilation of urinary tract. Urology 1980;16:266-9.

24. Colliver D, Storey R, Dickens H, et al. Nonobstructive urinary tract dilatation in children with diabetes insipidus. J Pediatr Surg 2012;47:752-5.

25. Yuksel OH, Kivrak M, Sahin A, et al. A case of idiopathic diabetes insipidus presented with bilateral hydroureteronephrosis and neurogenic bladder: A pediatric case report and literature review. Can Urol Assoc J 2015;9:E817-20.
Cite this article as: Sun L, Zhao D, Zhu L, Shen Y, Zhao Y, Tang D. Asymptomatic obstructive hydronephrosis associated with diabetes insipidus: a case report and review. Transl Pediatr 2021;10(6):1721-1727. doi: 10.21037/tp-20-476 